\title{
Fibroblast growth factor receptor 1 gene amplification is associated with poor survival in patients with resected esophageal squamous cell carcinoma
}

\author{
Hyo Song Kim ${ }^{1, *}$, Seung Eun Lee ${ }^{2, *}$, Yoon Sung Bae ${ }^{3, *}$, Dae Joon Kim ${ }^{4}$, Chang-Geol \\ Lee $^{5}$, Jin Hur6, Hyunsoo Chung7, Jun Chul Park7, Da Hyun Jung7, Sung Kwan Shin, \\ Sang Kil Lee ${ }^{7}$, Yong Chan Lee?, Hye Ryun Kim${ }^{1}$, Yong Wha Moon ${ }^{1}$, Joo Hang Kim ${ }^{1}$, \\ Young Mog Shim ${ }^{8}$, Susan S. Jewell ${ }^{9}$, Hyunki Kim ${ }^{3}$, Yoon-La Choi ${ }^{2}$ and Byoung Chul \\ Cho $^{1}$ \\ ${ }^{1}$ Division of Medical Oncology, Department of Internal Medicine, Yonsei University College of Medicine, Seoul, Korea \\ 2 Departments of Pathology, Samsung Medical Center, Sungkyunkwan University School of Medicine, Seoul, Korea \\ ${ }^{3}$ Department of Pathology, Yonsei University College of Medicine, Seoul, Korea \\ ${ }^{4}$ Department of Thoracic and Cardiovascular Surgery, Yonsei University College of Medicine, Seoul, Korea \\ ${ }^{5}$ Department of Radiation Oncology, Yonsei University College of Medicine, Seoul, Republic of Korea \\ ${ }^{6}$ Department of Radiology, Yonsei University College of Medicine, Seoul, Republic of Korea \\ ${ }^{7}$ Division of Gastroenterology, Department of Internal Medicine, Yonsei University College of Medicine, Seoul, Korea \\ 8 Department of Thoracic Surgery, Samsung Medical Center, Sungkyunkwan University School of Medicine, Seoul, Korea \\ ${ }^{9}$ Abbott Molecular Laboratories, Des Plaines, IL \\ * These authors contributed equally to this work as co-first authors \\ Correspondence to: Byoung Chul Cho, email: cbc1971@yuhs.ac \\ Yoon-La Choi, email: ylachoi@skku.edu \\ Hyunki Kim, email: kimhyunki@yuhs.ac
}

Keywords: Fibroblast growth factor receptor 1, esophageal squamous cell carcinoma, gene amplification, fluorescent in situ hybridization, prognostic factor

Received: September 11,2014 Accepted: December 09, $2014 \quad$ Published: December 10, 2014

This is an open-access article distributed under the terms of the Creative Commons Attribution License, which permits unrestricted use, distribution, and reproduction in any medium, provided the original author and source are credited.

\section{ABSTRACT}

To investigate the frequency and the prognostic impact of fibroblast growth factor receptor 1 (FGFR1) gene amplification in 526 curatively resected esophageal squamous cell carcinoma (ESCC). Using fluorescent in situ hybridization, high amplification was defined by an FGFR1/centromer 8 ratio is $\geq 2.0$, or average number of FGFR1 signals/ tumor cell nucleus $\geq 6.0$, or percentage of tumor cells containing $\geq 15$ FGFR1 signals or large cluster in $\geq 10 \%$. Low amplification was defined by $\geq 5$ FGFR1 signals in $\geq$ $\mathbf{5 0 \%}$. FGFR2 and FGFR3 mutations were assessed by direct sequencing in $\mathbf{3 8 8}$ cases and no mutation was detected. High and low amplification were detected in $8.6 \%$ and $1.1 \%$, respectively. High FGFR1 amplification had significantly shorter disease-free survival (34.0 vs 158.5 months $P=0.019$ ) and overall survival (52.2 vs not reached $P=0.022$ ) than low/no amplification group. After adjusting for sex, smoking, stage, histology, and adjuvant treatment, high FGFR1 amplification had a greater risk of recurrence (adjusted hazard ratio $[A H R], 1.6 ; P=0.029)$ and death (AHR, 1.53; $P=0.050$ ). High amplification was significantly higher in current smokers than former and never-smokers $\left(P_{\text {trend }}<0.001\right)$ and increased proportional to smoking dosage. High FGFR1 amplification is a frequent oncogenic alteration and an independent poor prognostic factor in resected ESCC. 


\section{INTRODUCTION}

Esophageal cancer (EC) is the sixth most common cause of cancer death worldwide [1]. Despite the improvement of surgical technique and medical treatment, prognosis remains poor, with 5-year survival rate of less than 40\%.[2] EC consists of two major histologic subtypes; esophageal squamous cell carcinoma (ESCC) and adenocarcinoma (EAC). Both histologic subtypes have different risk factors and epidemiology. ESCC continues to be the major subtype of EC in Asia, and the main risk factors include smoking and alcohol abuse.[3] In contrast, EAC predominately affects the Caucasian, and is closely associated with gastroesophageal reflux disease, Barrett's esophagus, and obesity. [4-7] Compared with ESCC, EAC shows younger age onset, male predominance and frequent occurrence in distal third of the esophagus. [8] Given widely different epidemiological and clinical features, EAC and ESCC may represent distinct disease entities which may benefit from different therapeutic approaches.

Amplification and overexpression of human epidermal growth factor receptor 2 (HER2; also known as ERBB2) was more frequent in EAC than ESCC and there was a strong concordance of HER2 status in primary and metastatic cancer.[9] Therefore, various clinical trials evaluating the efficacy of HER2 targeting agents were reported for the patients with advanced esophageal and esophagogastric junction (EGJ) adenocarcinoma. ${ }^{8-10}$ In the phase II trials for HER2 amplified EGJ adenocarcinoma, lapatinib, a dual tyrosine kinase inhibitor of ERBB1 and HER2 demonstrated modest response.[10, 11] The therapeutic implication of HER2 protein overexpression or gene amplification has been demonstrated in the Trastuzumab for Gastric Cancer (ToGA) trial [12]. In this trial, addition of trastuzumab (anti-HER2 antibody) to standard chemotherapy significantly improved response rate, progression-free survival and overall survival compared with chemotherapy alone in HER2-positive advanced gastric and EGJ adenocarcinoma. Based on the results of the ToGA trial, HER2 testing is routinely recommended for all patients with metastatic gastric or EGJ adenocarcinoma, and trastuzumab should be considered for HER2-positive cases.

Despite a rapid and enthusiastic development of targeted therapies in gastric or EGJ adenocarcinoma, no therapeutically tractable target has been identified for ESCC. Recently, comparative genomic analysis showed different DNA copy number alterations between EAC and ESCC.[13, 14] Among those, high copy number gains of cancer-associated genes, such as SOX2, PIK3CA, CCND1, and $F G F R 1$, were more frequently observed in ESCC than in $\mathrm{EAC}$, suggesting that genomic gain of these oncogenes may be therapeutic targets for ESCC.

Fibroblast growth factor receptor 1 (FGFR1) is a member of family of receptor tyrosine kinases (FGFR1-
4), and its activation by amplification, mutation, or translocation leads to tumor cell proliferation and survival in many cancers.[15, 16] Potentially actionable FGFR rearrangements were identified in diverse solid tumors including lung cancer, oral cancer, and breast cancer. [1719] FGFRI amplification has been frequently reported in lung squamous cell carcinoma ( $\mathrm{SqCC})$, small cell lung cancer, and $\mathrm{SqCC}$ of head and neck (SCCHN), for which smoking is a clear and dominant risk factor.[15, 16, 20-28] Overall, the frequency of FGFR1 amplification was reported to be $5.6 \%-24.8 \%$ in lung SqCC and $15 \%$ $17.4 \%$ in SCCHN, suggesting that this genetic alteration mainly target squamous cell histology.[16, 20-25, 27, 28] Furthermore, FGFR1 amplification has been associated with poor prognosis or unfavorable clinicopathologic parameters in lung SqCC and SCCHN. Because ESCC has risk factors in common with lung SqCC and SCCHN, we hypothesized that FGFR1 amplifications is associated with pathogenesis and poor prognosis in ESCC.

In this study, we sought to determine the frequency, prognostic impact and association with smoking dosage of FGFR1 amplification in surgically resected ESCC. Furthermore, we also evaluated the frequency of FGFR2 and FGFR 3 mutations in ESCC.

\section{RESULTS}

\section{Patient Characteristics}

A total of 526 Korean patients who underwent curative esophagectomy were analyzed. The clinical characteristics of the enrolled patients are presented in Table 1. There were 489 male and 37 female with a median age of 66 years (range 35-98). Median tumor size was $3 \mathrm{~cm}$ and approximately half of tumors had pT3 or pN0. Pathologic stages were I in $22.4 \%$, II in $42.8 \%$, and III in $34.8 \%$. Two thirds $(60.3 \%)$ were located in the lower esophagus, and more than half of patients had moderately differentiated SqCCs. The majority of patients were current $(39.0 \%)$ or former smokers $(38.6 \%)$, and median smoking dosage was 30.0 pack-years (range 0-150). Adjuvant treatment was given in 140 patients $(26.6 \%)$, and 87 of those $(62.1 \%)$ were treated by concurrent chemoradiotherapy. The proportions of adjuvant therapy according to stage were $5.9 \%$ in stage I, $21.8 \%$ in stage II, and $45.9 \%$ in stage III.

\section{FGFR1 amplification status and clinicopathologic features}

Among 526 patients, 45 (8.6\%) were high FGFR1 amplification, $6(1.1 \%)$ were low FGFR1 amplification, and 475 (90.3\%) displayed no amplification (Table 1; Figure 1). The median FGFR1 gene copy number per 
Table 1: Patient characteristics according to FGFR1 amplification

\begin{tabular}{|c|c|c|c|c|c|c|c|c|c|}
\hline \multirow[t]{2}{*}{ Characteristics } & \multicolumn{2}{|c|}{ All patients } & \multicolumn{2}{|c|}{ High amplification" } & \multicolumn{2}{|c|}{ Low amplification" } & \multicolumn{2}{|c|}{ No amplification } & \multirow[b]{2}{*}{$\boldsymbol{P}^{\dagger}$} \\
\hline & No. & $\%$ & No. & $\%$ & No. & $\%$ & No. & $\%$ & \\
\hline No of patients & 526 & 100 & 45 & 8.6 & 6 & 1.1 & 475 & 90.3 & \\
\hline Age, years & & & & & & & & & 0.697 \\
\hline Median (range) & \multicolumn{2}{|c|}{$66(35-98)$} & \multicolumn{2}{|c|}{$67(52-77)$} & \multicolumn{2}{|c|}{$68(65-77)$} & \multicolumn{2}{|c|}{$66(35-98)$} & \\
\hline Sex & & & & & & & & & 0.608 \\
\hline Male & 489 & 93.0 & 43 & 95.6 & 6 & 100 & 440 & 92.6 & \\
\hline Female & 37 & 7.0 & 2 & 4.4 & 0 & 0 & 35 & 7.4 & \\
\hline Tumor size, $\mathrm{cm}$ & & & & & & & & & 0.310 \\
\hline Median, range & \multicolumn{2}{|c|}{$3.0(0.2-10.5)$} & \multicolumn{2}{|c|}{$3.0(2.0-9.0)$} & \multicolumn{2}{|c|}{$2.5(2.0-4.5)$} & \multicolumn{2}{|c|}{$3.0(0.2-10.5)$} & \\
\hline pT stage & & & & & & & & & 0.618 \\
\hline $\mathrm{T} 1$ & 167 & 31.7 & 12 & 26.7 & 2 & 33.3 & 153 & 32.2 & \\
\hline $\mathrm{T} 2$ & 106 & 20.2 & 12 & 26.7 & 1 & 16.7 & 93 & 19.6 & \\
\hline $\mathrm{T} 3$ & 238 & 45.2 & 18 & 40.0 & 3 & 50.0 & 217 & 45.7 & \\
\hline $\mathrm{T} 4$ & 15 & 2.9 & 3 & 6.7 & 0 & 0 & 12 & 2.5 & \\
\hline pN stage & & & & & & & & & 0.619 \\
\hline No & 259 & 49.2 & 22 & 48.9 & 3 & 50.0 & 234 & 49.3 & \\
\hline N1 & 234 & 44.5 & 20 & 44.4 & 2 & 33.3 & 212 & 44.6 & \\
\hline $\mathrm{N} 2$ & 22 & 4.2 & 3 & 6.7 & 1 & 16.7 & 18 & 3.8 & \\
\hline N3 & 11 & 2.1 & 0 & 0 & 0 & 0 & 11 & 2.3 & \\
\hline pTMN stage & & & & & & & & & 0.822 \\
\hline I & 118 & 22.4 & 8 & 17.8 & 1 & 16.7 & 109 & 22.9 & \\
\hline II & 225 & 42.8 & 19 & 42.2 & 2 & 33.3 & 204 & 42.9 & \\
\hline III & 183 & 34.8 & 18 & 40.0 & 3 & 50.0 & 162 & 34.1 & \\
\hline Location & & & & & & & & & 0.158 \\
\hline Upper & 66 & 12.5 & 5 & 11.1 & 0 & 0 & 61 & 12.8 & \\
\hline Middle & 143 & 27.2 & 19 & 42.2 & 2 & 33.3 & 122 & 25.7 & \\
\hline Lower & 317 & 60.3 & 21 & 46.7 & 4 & 66.7 & 292 & 61.5 & \\
\hline Tumor grade & & & & & & & & & 0.424 \\
\hline Well & 128 & 24.3 & 12 & 26.7 & 2 & 33.3 & 114 & 24.0 & \\
\hline Moderate & 314 & 59.7 & 29 & 64.4 & 4 & 66.7 & 281 & 59.2 & \\
\hline Poorly & 84 & 16.0 & 4 & 8.9 & 0 & 0 & 80 & 16.8 & \\
\hline Smoking status & & & & & & & & & $<0.001$ \\
\hline Never-smoker & 118 & 22.4 & 1 & 2.2 & 0 & 0 & 117 & 24.6 & \\
\hline Former smoker & 203 & 38.6 & 5 & 11.1 & 2 & 33.3 & 196 & 41.3 & \\
\hline Current smoker & 205 & 39.0 & 39 & 86.7 & 4 & 66.7 & 162 & 34.1 & \\
\hline \multicolumn{10}{|c|}{$\begin{array}{l}\text { Smoking dosage, pack- } \\
\text { years }\end{array}$} \\
\hline Median & \multicolumn{2}{|l|}{30} & \multicolumn{2}{|l|}{39} & \multicolumn{2}{|l|}{35} & \multicolumn{2}{|l|}{30} & 0.002 \\
\hline Range & \multicolumn{2}{|l|}{$0-150$} & \multicolumn{2}{|l|}{$0-99$} & \multicolumn{2}{|c|}{$13-60$} & $0-150$ & & \\
\hline Adjuvant therapy & & & & & & & & & 0.870 \\
\hline Yes & 140 & 26.6 & 13 & 28.9 & 2 & 33.3 & 125 & 26.3 & \\
\hline No & 386 & 73.4 & 32 & 71.1 & 4 & 66.7 & 350 & 73.7 & \\
\hline FGFR1 $\mathrm{FISH}^{\S}$ & & & & & & & & & \\
\hline $\begin{array}{c}\text { Number } \\
\text { (median, range) }\end{array}$ & $2.2(0$ & & 6.4 & 5.5) & $5.1(5$ & & $2.2(0$ & & $<0.001$ \\
\hline $\begin{array}{c}\text { Ratio } \\
\text { (mean, range) }\end{array}$ & $1.5(0$ & & 2.9( & & $1.5(1$ & & $1.3(0$ & & 0.003 \\
\hline
\end{tabular}


Abbreviations:

"High FGFR1 amplification was defined as if one of the following criteria is fulfilled: (1) FGFR1/CEN8 ratio is $\geq 2.0$, (2) average number of $F G F R 1$ signal per nucleus $\geq 6.0$, and (3) percentage of tumor cells containing $\geq 15 F G F R 1$ signals or large clusters in $\geq 10 \%$ cells. Low FGFR 1 amplification was defined when the percentage of tumor cells containing $\geq 5$ FGFR 1 signals is $\geq 50 \%$.

${ }^{\dagger} \chi^{2}$ test, Fisher's exact test, or Mann-Whitney U test.

*Pathologic stage at the time of surgical resection was determined according to the American Joint Committee on Cancer (seventh edition) guidelines.

"Never-smokers; a lifetime smoking dose of fewer than 100 cigarettes; former smokers, those who have stopped smoking for more than 1 year; current smokers, those who currently smoke or have quit for less than 1 year.

${ }^{\S} F G F R 1$ numbers are average numbers of FGFR1 signals per nucleus, and ratios are FGFR1/CEN8 ratios.

nucleus and the mean FGFR1/CEN8 ratio in all patients were 2.2 (range, 0 to 15.5 copies per nucleus) and 1.5 (range, 0 to 7.8). The median FGFR1 gene copy number was 6.4 (range, 4.1 to 15.5 ) in high amplification, 5.1 (range, 5.0 to 5.6) in low amplification, and 2.2 (range 0 to 5.7) in no amplification group. The mean FGFR1/ CEN8 ratio was 2.9 (range 1.1 to 7.8 ), 1.5 (range, 1.0 to 1.9 ), and 1.3 (range, 0 to 1.6 ) in high, low and no amplification group, respectively. Of 45 high FGFR amplified tumors, 12 cases $(26.7 \%)$ only satisfied the criterion of an FGFR1:CEN8 ratio of $\geq 2.0,7$ cases $(15.6 \%)$ only satisfied the criterion of an average number of FGFR 1 signal per nucleus $\geq 6.0$, and 4 cases $(8.9 \%)$ only satisfied the criterion of percentage of tumor cells containing $\geq 15$ FGFR 1 signals or large clusters in $\geq 10 \%$ cells, respectively. 22 cases $(48.9 \%)$ satisfied all three criteria for FGFR1 amplification simultaneously.

There was no significant difference among three FGFR groups regarding age, sex, tumor size, pathologic stage, location, histologic grade, and adjuvant therapy (Table 1). However, the incidence of high FGFRl amplification was significantly higher in smokers than in never-smokers $(\mathrm{P}<0.001)$. Only $1(2.2 \%)$ of 45 patients with high-level amplification was never smoker. The median smoking dosage was significantly higher for high amplification group than that for low or no amplification group (39 vs 35 vs 30 pack-years, $P=0.002$ ). Based on the pattern of high amplification (group fulfilling 3 criteria $v s$. 1 or 2 criteria), there was no significant difference in terms of age, sex, location, and pathologic stage.

\section{Survival Outcomes According to FGFR1 Amplification}

With a median follow-up time of 55.4 months, the 5 year DFS and OS rates for all patients were $52.8 \%$ and $59.8 \%$, respectively. The 5 -year DFS rate according to pTNM stages were $78.4 \%$ in stage I, $55.9 \%$ in stage II, and $33.2 \%$ in stage III patients. The 5-year OS rate according to pTNM stages were $86.8 \%$ for stage I, $65.3 \%$ for stage II, and $37.1 \%$ for stage III.

The median DFS for each of the three FGFR groups was 34.0 months in the high FGFR1 amplification, not reached (NR) in low amplification group, and 158.5 months in the no amplification group (Figure 2A). By using pair-wise comparison, patients with high FGFRI amplification showed a significantly shorter DFS than those with no amplification (34.0 vs 158.5 months in no amplification, $P=0.020$ ). The median DFS of high amplification and low amplification were not significantly different (34.0 vs NR in low amplification, $P=0.272$ ), probably due to small sample size in the low amplification group. The median DFS was similar between low amplification and no amplification group in pair-wise
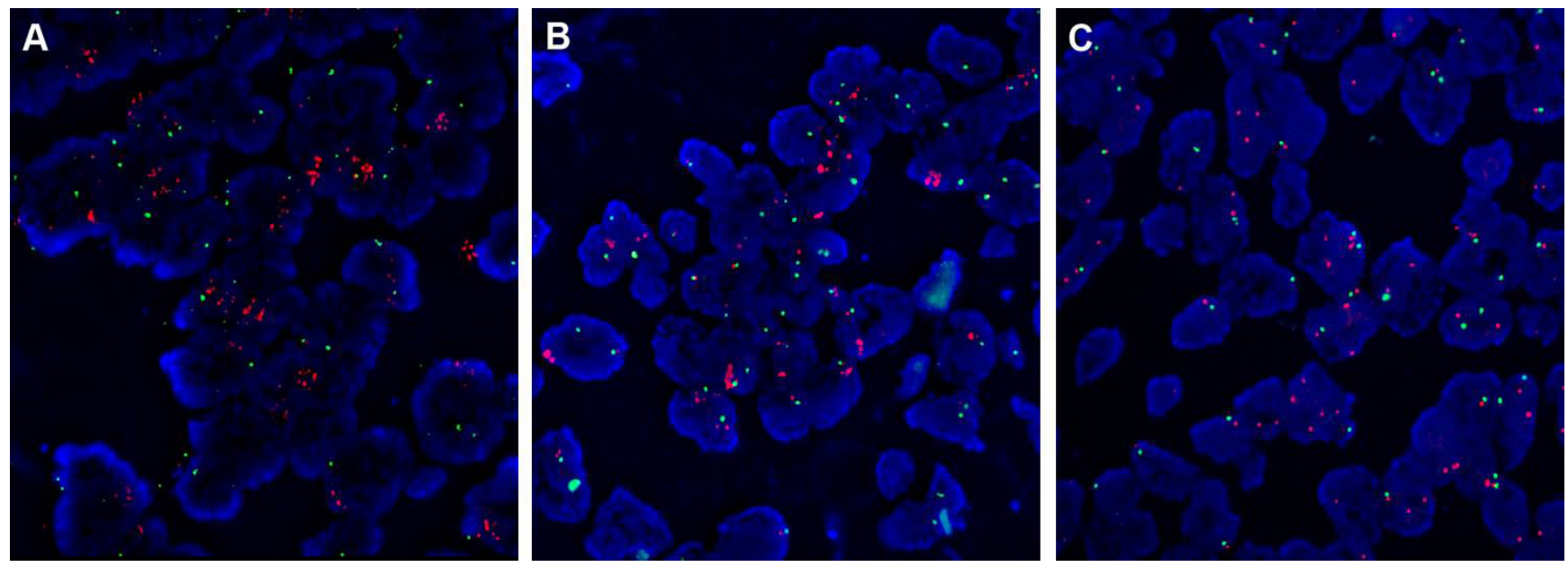

Figure 1: Fibroblast growth factor receptor 1 (FGFR1) amplification assessed by fluorescent in situ hybridization. (A) High FGFR1 amplification; (B) Low FGFR1 amplification; (C) No amplification. 
Table 2: Survival outcome in multivariate analysis

\begin{tabular}{|l|l|l|l|l|l|l|l|}
\hline & \multicolumn{3}{|l|}{ DFS } & \multicolumn{2}{l|}{ OS } \\
\hline Variable & Category & HR & $\mathbf{9 5 \%}$ CI & P & HR & 95\% CI & P \\
\hline Sex & $\begin{array}{l}\text { Male vs } \\
\text { female (ref) }\end{array}$ & 0.91 & $0.53-1.55$ & 0.724 & 0.90 & $0.53-1.54$ & 0.703 \\
\hline Smoking status & $\begin{array}{l}\text { Current smoker vs } \\
\text { never/former-smoker } \\
\text { (ref) }\end{array}$ & 1.08 & $0.82-1.43$ & 0.595 & 1.02 & $0.77-1.35$ & 0.898 \\
\hline Pathologic stage* & III vs II/I (ref) & 2.56 & $1.97-3.34$ & $<0.001$ & 2.53 & $1.94-3.29$ & $<0.001$ \\
\hline Histology & $\begin{array}{l}\text { Poor vs well/ } \\
\text { moderate(ref) }\end{array}$ & 1.61 & $1.16-2.22$ & 0.004 & 1.61 & $1.17-2.23$ & 0.004 \\
\hline Adjuvant treatment & Yes vs no (ref) & 1.11 & $0.82-1.50$ & 0.497 & 1.05 & $0.78-1.43$ & 0.731 \\
\hline FGFR1 amplification & $\begin{array}{l}\text { High vs (ref) } \\
\text { Low /no (ref) }\end{array}$ & 1.61 & $1.05-2.46$ & 0.029 & 1.53 & $0.99-2.34$ & 0.050 \\
\hline
\end{tabular}

Abbreviations: DFS, disease-free survival; OS, overall survival; ref, reference; amp, amplification;

* Clinical stage at the time of initial diagnosis was determined according to the American Joint Committee on Cancer (seventh edition) guidelines.

comparison $(P=0.639)$. The median OS for each of the three FGFR groups was 52.2 months in the high FGFRI amplification, 72.0 months in the low amplification, and not reached in the no amplification (Figure 2B). By using pair-wise comparison, patients with high FGFRl amplification showed a significantly shorter OS than those with no amplification (52.2 vs NR in no amplification, $P=0.021)$. The OS times of high amplification and low amplification were not significantly different (52.2 vs
(A)

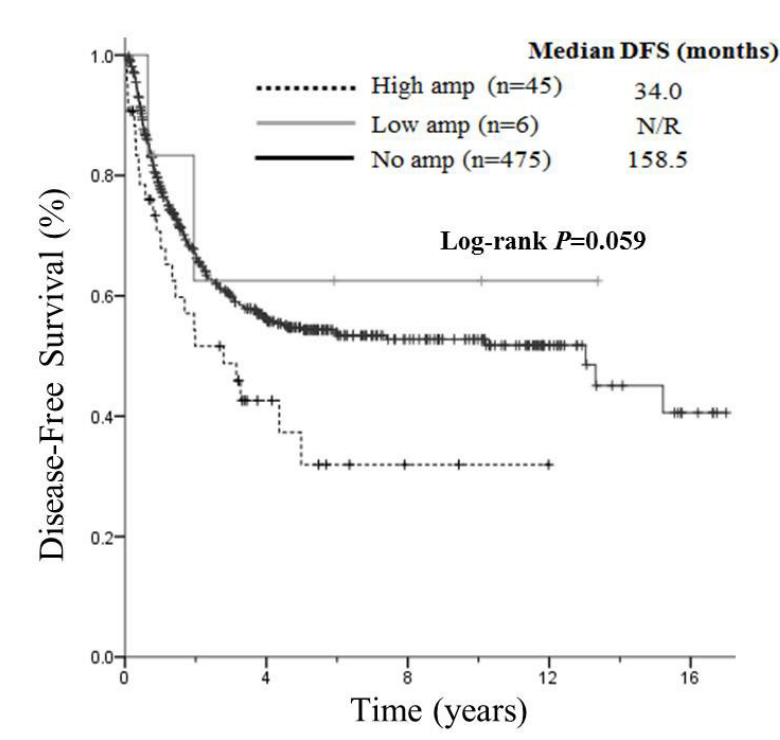

72.0 in low amplification, $P=0.637$ ), probably due to small sample size in the low amplification group. The median OS was similar between low amplification and no amplification group in pair-wise comparison $(P=0.517)$.

Because the DFS of patients with low and no amplification were similar but different from that of patients with high amplification, we categorized total patients into high amplification group and low/no amplification group and assessed the survival outcomes
(B)

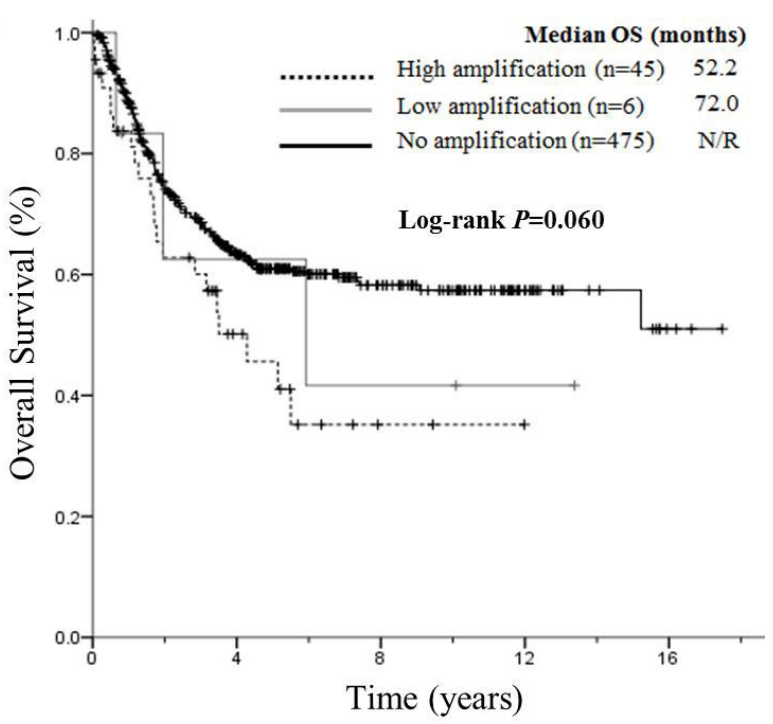

Figure 2: Survival analysis on the basis of FGFR1 amplification (high, low, and no amplification). (A) Median DFS was 34.0 months in the high FGFR1 amplification group, not reached in low amplification group, and 158.5 months in the no amplification group. (B) The median OS was 52.2 months in the high FGFR1 amplification group, and 72.0 months in the low amplification group, and not reached in the no amplification group. 
of these two groups. The median DFS of the high FGFR1 amplification group was significantly shorter compared with that of the low/no FGFR1 amplification group (34.0 vs 158.5 months $P=0.019$, Figure $3 \mathrm{~A}$ ). In addition, high FGFR1 amplification group demonstrated significantly shorter OS than low/no FGFR1 amplification group (52.2 vs NR, $P=0.022$, Figure $3 \mathrm{~B}$ ). In Cox proportional hazard model adjusted for sex, smoking history, pathologic stage, adjuvant treatment, and histologic grade, high FGFR1 amplification was significantly associated with a shorter DFS (AHR 1.61; 95\% CI, 1.05-2.46; $P=0.029$, table 2). There was a strong trend toward worse OS for high FGFRl amplification compare to low/no FGFR1 amplification group in multivariate analysis (HR 1.53; 95\% CI, 0.99-2.34, $P=0.050$ ). There was no significant difference in DFS and OS for sex, smoking status, and adjuvant treatment in multivariate analysis. There was no significant difference in DFS and OS between group fulfilling 3 criteria and group fulfilling 1 or 2 criteria.

\section{FGFR2 and FGFR3 mutations}

FGFR2 and FGFR3 mutations were assessed by direct sequencing in 388 cases with FFPE tissues available and no FGFR 2 or FGFR3 mutation was detected.
(A)

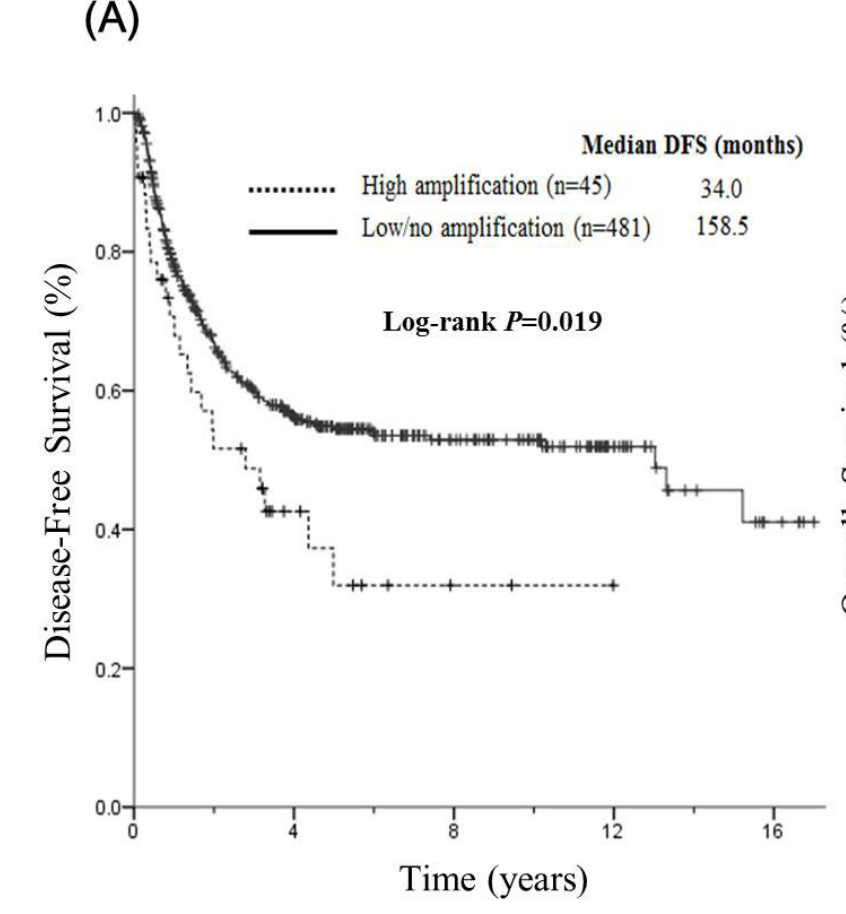

\section{Association between $F G F R 1$ amplification and} smoking

As shown in Figure 4A, the incidence of high-level FGFR1 amplification was significantly higher in current smokers than former or never-smokers $\left(P_{\text {trend }}<0.001\right)$. The incidences of high-level FGFR1 amplification in current, former and never-smokers were $19.0 \%, 2.5 \%$, and $0.8 \%$, respectively. With increment of total cigarette smoking dosage, the incidence of high-level FGFR1 amplification was significantly increased (Figure 4B, $P_{\text {trend }}=0.001$ ). High-level FGFR1 amplification were $0.8 \%$ for neversmokers, $5.8 \%$ in patients with $1-10$ pack-years, $7.4 \%$ with $11-20$ pack-years, $9.6 \%$ with $21-50$ pack-years, and $19.3 \%$ with more than 50 pack-years.

\section{DISCUSSION}

In this study, we investigated the frequency and the prognostic impact of FGFR1 amplification in resected ESCC. To our knowledge, this is the first report on the prognostic impact of FGFR1 amplification in the largestever cohort of resected ESCC patients from East Asian. Our study demonstrated high FGFRl amplification is a common genetic alteration $(8.6 \%)$ and an independent negative prognostic factor in resected ESCC. Interestingly, it was associated with cigarette smoking in a dosedependent manner.
(B)

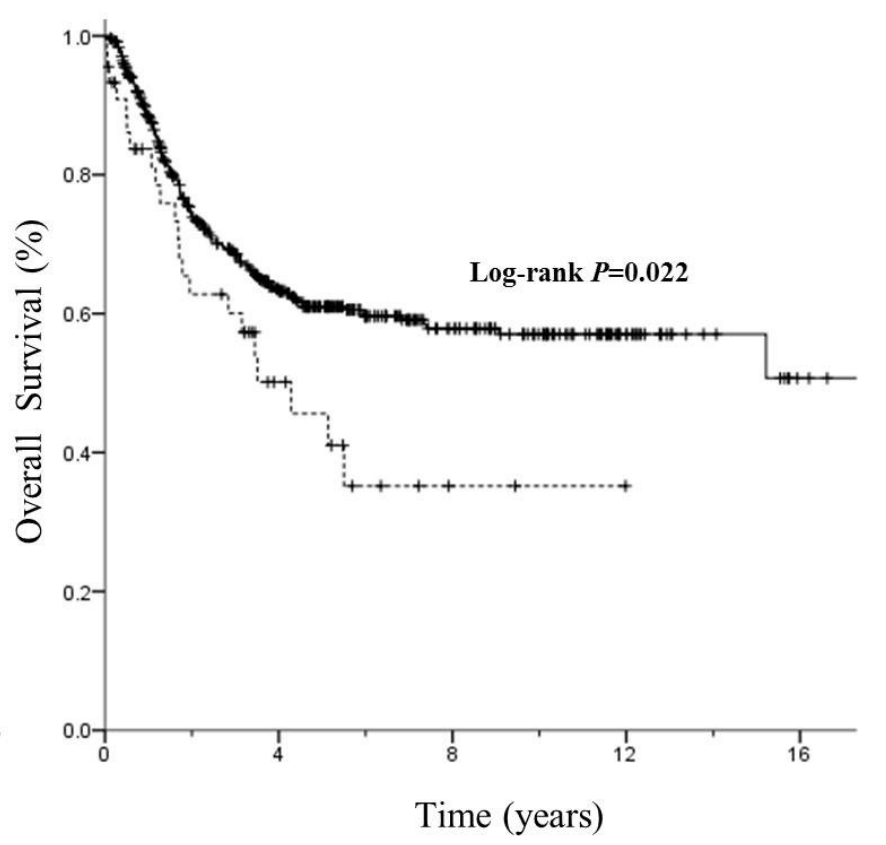

Figure 3: Prognostic impact of high FGFR1 amplification and low/no amplification on DFS and OS. (A) The median DFS of the high FGFR1 amplification group was significantly shorter compared with low/no $F G F R 1$ amplification group $(P=0.019)(\mathrm{B})$ The median OS of high FGFR1 amplification group demonstrated significantly shorter than low/no $F G F R 1$ amplification group $(P=0.022)$. 
The frequency of FGFR1 amplification has been reported to range from $6 \%$ to $9.4 \%$. In the DNA array hybridization study,[29] 2 out of 32 ESCC cases (6\%) demonstrated amplified 8p11 locus which containing FGFR1 gene. A comparative genomic study revealed many focal DNA amplifications or losses such as $S O X 2$, PIK3CA, CCND1, FGFR1, MYC, GATA4, and GATA6 in ESCC and EAC.[13] Of note, FGFR1 was amplified in $21 \%$ of ESCC samples compare with $8 \%$ in EAC. Recently, FGFR1 amplification was reported to be $9.4 \%$ in the cohort with Western Europe.[30] The frequency of high FGFR1 amplification (8.6\%), as determined by FISH analysis in our study, was comparable to those reported in previous studies.[13, 29, 30] Given the relatively high frequency of FGFR1 amplification, it may represent an attractive therapeutic target for ESCC.

Given the histological similarity and common risk factors, it is not surprising that FGFR1 amplification has been reported in upper aerodigestive tract cancers such as lung SqCC, SCCHN, and small cell lung cancer. [16, 20-28] However, the prognostic significance of FGFR1 amplification in these cancers has shown controversial results. In resected lung SqCC, Kim et al[16] reported FGFR1 amplification as negative prognostic factor, whereas Heist et al [24] observed no significant difference in OS. In SCCHN, FGFRI amplification was significantly associated with poor prognostic factors such as higher T stage, lymphovascular invasion, and higher numbers of visceral metastases.[22] Tumor heterogeneity, unstandardized FISH criteria for FGFR1 amplification, varying adjuvant treatment, and small sample size may contribute to the controversial results. In our study, by applying more sophisticated criteria for FGFR1 amplification, we were able to divide FGFR1 amplification into high and low amplification.
Low FGFR1 amplification occurred only in $1.1 \%$ and survival outcome of low FGFR1 amplification group was similar to no amplification group. Compared with low/ no amplification group, high FGFRl amplification was significantly associated with shorter DFS regardless of sex, histology, and adjuvant therapy, implying that FGFR 1 amplification as an independent negative prognostic factor in curatively resected ESCC.

The identification of FGFR alteration in human cancers led to rapid development of selective FGFR inhibitor such as BGJ398 and AZD4547. In phase I study with BGJ398,[31] 1 patient with FGFR1 amplified lung SqCC with $F G F R 1 / C E N 8$ ratio of 2.6 by FISH analysis had partial response. In another phase I study with FGFR inhibitor AZD4547,[32] 1 partial response was also seen in lung SqCC patient with FGFR1 amplification (FGFR1/ CEN8 ratio $>2.8$ ). Various FGFR inhibitors are currently in clinical development for the patients with FGFR aberrations. Therefore, our study strongly suggests the therapeutic potential of FGFR inhibitor for ESCC, and future clinical trials for advanced high FGFR1 amplified ESCC are strongly warranted.

Unfortunately, FISH criteria for FGFR1 amplification has not yet been standardized. Indeed, definition of FGFR1 amplification by FISH technique has been highly variable in the previous studies in lung cancer and breast cancer.[16, 24, 25, 28, 33, 34] Unlike breast cancer, lung SqCC exhibits small-clusters and co-amplifications of FGFR1 and CEN8.[25] Therefore, FGFR1 FISH assay needs to differentiate between true amplification and polysomy. In a large cohort study, Schildhause et al [25] proposed a more sophisticated FGFR1 FISH criteria using average gene copy number per nucleus, $F G F R 1 / \mathrm{CEN} 8$ ratio, and percentage of gene clusters at the same time. By the addition of FGFR1/CEN8
(A)

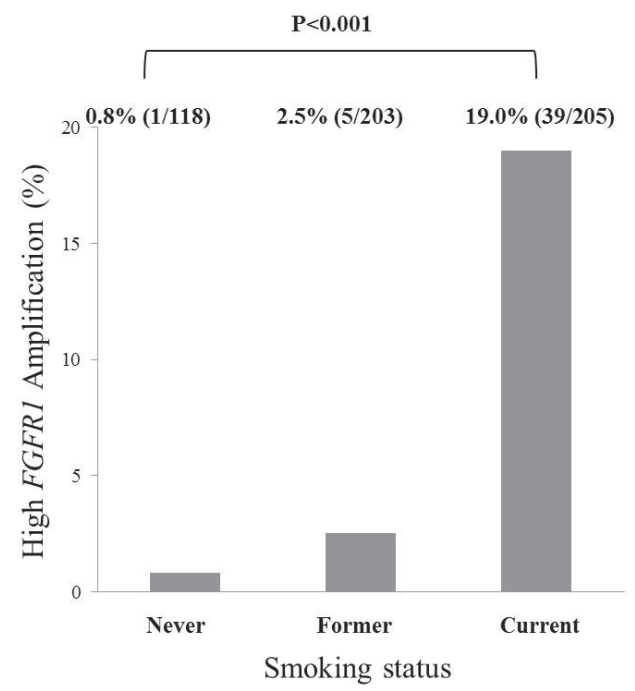

(B)

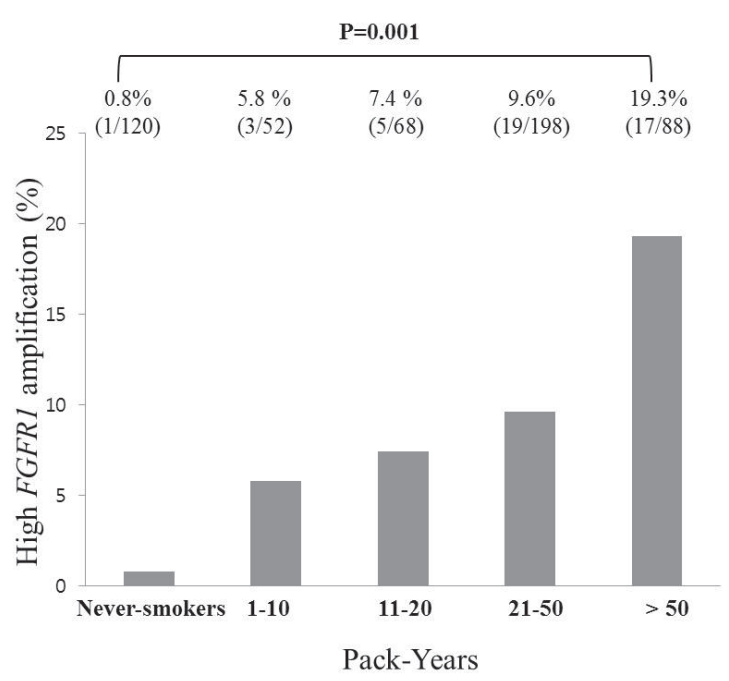

Figure 4: Association of smoking status and high FGFR1 amplification. (A) Proportions of high $F G F R 1$ amplification according to never-, former, and current smokers (B) Incidence of high FGFRl amplification according to smoking dosage. 
ratio, 8 out of 47 cases $(17.0 \%)$ were newly classified as high amplification in this study.(25) Similarly, in our study, 12 out of 45 high amplification group (26.7\%) might have been misclassified as low/no amplification group if the criterion for $F G F R 1 / C E N 8$ ratio was not included. Considering clinical benefit appeared in patients with high $F G F R 1 / C E N 8$ ratio in clinical trials with FGFR inhibitors, [31, 32] this scoring system might serve as a standardized screening tool to select patients who gain greater benefit from treatment with FGFR inhibitors. Our criteria need further validation in the future clinical trials with FGFR inhibitors in ESCC patients.

Accumulating evidence has shown that FGFRl amplification correlated with smoking status in squamous cell biology. FGFR1 amplification occurs significantly more often in the smokers of the lung SqCC, SCCHN, and small cell lung cancer.[16, 22, 23, 26] The proportion of FGFR1 amplification among current smokers were reported $15.8 \%$ to $28.9 \%$ in lung SqCC and $17.7 \%$ in SCCHN in a dose dependent manner.[16, 22, 23] In small cell lung cancer cohort, all FGFR1 amplified cases were current or former smokers.[26] In our study, FGFRI amplification was significantly more likely to be smokers, and 44 out of 45 high FGFR1 amplification cases were current or former smokers. Similar to the findings in lung SqCC and SCCHN, acquisition of FGFR1 amplification in our study was also significantly increased proportional to smoking dosage. Therefore, FGFRl amplification may be an oncogenic driver mutation in tobacco-associated cancers of the aerodigestive tract. An interesting question of the role of FGFR1 amplification on the smokingassociated carcinogenesis still remained to be solved.

Somatic mutations of FGFR2 and FGFR3 were reported in $12 \%$ and $30 \%$ of endometrial and urothelial cell carcinomas, respectively.[35, 36] Inhibitor-sensitive 5 mutation loci for $F G F R 2$ and 6 mutation loci for FGFR3 were noticed in 3\% of lung SqCC samples.[37] Here, we show no FGFR2 and FGFR 3 mutations in our 388 ESCC patients. This may be explained by the limited sensitivity of the Sanger sequencing, tumor heterogeneity or molecular difference of ESCC from lung SqCC.

The main limitation of our study include its retrospective nature and patient selection. Therefore, our findings should be validated in an independent cohort and response data to FGFR-targeted therapies in the future clinical trials.

In conclusion, we demonstrated high FGFRI amplification is an independent poor prognostic factor in resected ESCC. Patients with FGFR1 amplification were significantly more likely to be smokers and the frequency of FGFR 1 amplification was also increased proportional to smoking dosage, suggesting FGFRl amplification as an oncogenic aberration induced by smoking carcinogen. Our finding indicates FGFR1 amplification is a promising therapeutic target in ESCC.

\section{PATIENTS AND METHODS}

\section{Patients and tissue samples}

This study was conducted in a cohort of patients with ESCC who underwent radical esophagectomy at Severance Hospital and Samsung Medical Center, Seoul, Korea, between 2002 and 2010. The criteria used for patient selection included (1) surgically resected SqCC of the thoracic esophagus (R0 resection), (2) availability of primary tumor tissue, (3) no distant metastasis, and (4) no preoperative treatment. The tumor samples of 664 patients were available for examination of FGFR1 amplification. We excluded 115 cases $(17.3 \%)$ who received neoadjuvant treatment. Twenty-three patients $(3.5 \%)$ were excluded because of incomplete survival follow-up and/or smoking data. All diagnosis were reviewed by two experienced pathologist (Y.L.C. and H.K.K) and confirmed by hematoxylin and eosin staining. Three representative cores measuring in 2-mm-diameter for each paraffin-embedded primary tumor were assembled into tissue microarray blocks.

Patients' information was collected by reviewing the medical records for evaluation of clinicopathologic characteristics and survival outcome. Staging was determined using the $7^{\text {th }}$ edition American Joint Committee on Cancer guideline of tumor, node, and metastasis (TNM) classification. Smoking status such as never-smoker, former smoker, and current smoker were defined as previous studies.[38] The study was approved by the institutional review board of Severance Hospital and Samsung Medical Center.

\section{FGFR1 Fluorescence In Situ Hybridization}

Fluorescence in situ hybridization (FISH) to detect FGFR1 amplification was performed at the chromosomal level of the tissue microarray. We performed fluorescence signal detection with two probes on chromosome 8 . The target probe is located on the FGFR1 locus spanning 8 p12 - 8p11.23 with spectrum orange (red) and CEN 8 with Spectrum Green (Abbott Molecular, Abbott Park, IL) following routine method. The evaluation was done independently by two experienced pathologists (Y.L.C. and H.K.) blinded to clinical information, and at least 100 nuclei per case were evaluated. FGFR1 amplification was defined based on the previous studies.[25, 26] High FGFR1 amplification was defined as if one of the following criteria is fulfilled: (1) $F G F R 1 / \mathrm{CEN} 8$ ratio is $\geq 2.0$, (2) average number of FGFR1 signal per nucleus $\geq 6.0$, and (3) percentage of tumor cells containing $\geq$ 15 FGFR 1 signals or large clusters in $\geq 10 \%$ cells. Low FGFR1 amplification was defined when the percentage of tumor cells containing $\geq 5$ FGFR 1 signals is $\geq 50 \%$. 


\section{Mutation analysis}

Genomic DNA was extracted from 388 formalinfixed paraffin-embedded (FFPE) tissue specimens using an QIAamp DNA Micro kit (Qiagen, CA, USA) according to the manufacturer's instruction. The extracted DNA was used in a PCR amplification reaction. Based on the previous study in lung SqCC,[37] we evaluated the W290C, S320C, and K660E/K660N mutations in FGFR2, and $\mathrm{R} 248 \mathrm{C}$ and $\mathrm{S} 249 \mathrm{C}$ mutations in FGFR3. PCR amplification primers were designed to amplify following regions; W290C; 5'-TCCACAGTGGTCGGAGGAG-3'; 5'- $\quad$ AAAGTCCTCACCTTGAGAACCTTG-3', S320C; 5'-CCTGGTTGGCCGTTATATTG-3'; 5'- TGTTTTGGCAGGACAGTGAG-3', K660E/ K660N; 5'- ATTCATCGAGATTTAGCAGCCAG -3'; 5'- ACATTCTGAGCCTCACCCC -3', R248C and S249C; 5'-TGGCGGTGGTGGTGAG-3'; 5'ATTCACCTCCACGTGCTTGA-3'.

PCR was carried out with the following conditions : initial denaturation at $95^{\circ} \mathrm{C}$ for $10 \mathrm{~min}$, followed by 45 cycles for $30 \mathrm{~s}, 54^{\circ} \mathrm{C}$ for $60 \mathrm{~s}, 72^{\circ} \mathrm{C}$ for $45 \mathrm{~s}$ and final polymerization step of $72^{\circ} \mathrm{C}$ for $5 \mathrm{~min}$ in a GeneAmp PCR system 2720 (Life technologies, CA, USA). The amplified DNA product was visualized by gel electrophoresis and PCR products were sequenced using the Big dye terminator sequencing kit (Life technologies, CA, USA) according to the manufactures' instruction. Sequence reactions were the subjected to electrophoresis on an Applied Biosystems 3130XL DNA Analyzer (Life technologies, CA, USA).

\section{Statistical Analysis}

We evaluated association between FGFRI amplification status and clinical significance using the $\chi^{2}$ test or Fisher's exact test and trend was analyzed using linear regression. We also assessed whether FGFR1 amplification influenced survival outcome using KaplanMeier curves with a log-rank test. Disease free survival (DFS) was defined from the time of surgery to initial relapse or death. Overall survival (OS) was measured from the time of surgery to death or last follow-up date, and $95 \%$ CIs were evaluated by survival analysis using the Kaplan-Meier method. Statistical significance was set at $P<0.05$ for all analyses. Multivariate analysis was done using Cox regression analysis for following variables: sex, smoking status, stage, histology, adjuvant treatment, and FGFR1 status. All statistical analysis was performed using SPSS version 18.0 (SPSS, Chicago, IL).

\section{Disclosure of Potential Conflicts of Interest}

The authors have no potential conflicts of interest to disclose.

\section{ACKNOWLEDGEMENTS}

The FGFR1 FISH probe used in this study was supported by Abbott molecular Inc. (Abbott Park, IL).

\section{Grant}

This study was supported by a grant from the Korea Health Technology R\&D Project, Ministry of Health \& Welfare, Republic of Korea (HI12C1440, B. C. Cho) and by a faculty research grant of Yonsei University College of Medicine for 2012(6-2012-0044, H. Kim) and by the R\&D Program for Society of the National Research Foundation (NRF) funded by the Ministry of Science, ICT \& Future Planning (NRF-2013M3C8A1078501, Y. L. Choi).

\section{REFERENCES}

1. Kamangar F, Dores GM, Anderson WF. Patterns of cancer incidence, mortality, and prevalence across five continents: Defining priorities to reduce cancer disparities in different geographic regions of the world. Journal of Clinical Oncology 2006;24:2137-50.

2. Ando N, Ozawa S, Kitagawa Y, Shinozawa Y, Kitajima M. Improvement in the results of surgical treatment of advanced squamous esophageal carcinoma during 15 consecutive years. Ann Surg 2000;232:225-32.

3. Jemal A, Bray F, Center MM, Ferlay J, Ward E, Forman D. Global cancer statistics. CA Cancer J Clin 2011;61:69-90.

4. Wong A, Fitzgerald RC. Epidemiologic risk factors for Barrett's esophagus and associated adenocarcinoma. Clin Gastroenterol Hepatol 2005;3:1-10.

5. Sampliner RE. Epidemiology, pathophysiology, and treatment of Barrett's esophagus: reducing mortality from esophageal adenocarcinoma. Med Clin North Am 2005;89:293-312.

6. Layke JC, Lopez PP. Esophageal cancer: a review and update. Am Fam Physician 2006;73:2187-94.

7. Freeman HJ. Risk of gastrointestinal malignancies and mechanisms of cancer development with obesity and its treatment. Best Pract Res Clin Gastroenterol 2004;18:116775.

8. Siewert JR, Stein HJ, Feith M, Bruecher BLDM, Bartels H, Fink U. Histologic tumor type is an independent prognostic parameter in esophageal cancer: Lessons from more than 1,000 consecutive resections at a single center in the Western world. Annals of Surgery 2001;234:360-7.

9. Reichelt U, Duesedau P, Tsourlakis M, Quaas A, Link 
BC, Schurr PG, et al. Frequent homogeneous HER-2 amplification in primary and metastatic adenocarcinoma of the esophagus. Mod Pathol 2007;20:120-9.

10. Galsky MD, Von Hoff DD, Neubauer M, Anderson T, Fleming M, Nagarwala Y, et al. Target-specific, histologyindependent, randomized discontinuation study of lapatinib in patients with HER2-amplified solid tumors. Invest New Drugs 2012;30:695-701.

11. Hecht J, Urba S, Koehler M, Ellis C, Gagnon R, Kemner $A$, et al. Lapatinib monotherapy in recurrent upper gastrointestinal malignancy: phase II efficacy and biomarker analyses. Proc GI ASCO 2008;43.

12. Bang YJ, Van Cutsem E, Feyereislova A, Chung HC, Shen L, Sawaki A, et al. Trastuzumab in combination with chemotherapy versus chemotherapy alone for treatment of HER2-positive advanced gastric or gastro-oesophageal junction cancer (ToGA): a phase 3, open-label, randomised controlled trial. Lancet 2010;376:687-97.

13. Bandla S, Pennathur A, Luketich JD, Beer DG, Lin L, Bass AJ, et al. Comparative Genomics of Esophageal Adenocarcinoma and Squamous Cell Carcinoma. Annals of Thoracic Surgery 2012;93:1101-6.

14. Rumiato E, Pasello G, Montagna M, Scaini MC, De Salvo GL, Parenti A, et al. DNA copy number profile discriminates between esophageal adenocarcinoma and squamous cell carcinoma and represents an independent prognostic parameter in esophageal adenocarcinoma. Cancer Letters 2011;310:84-93.

15. Kim HS, Mitsudomi T, Soo RA, Cho BC. Personalized therapy on the horizon for squamous cell carcinoma of the lung. Lung Cancer 2013;80:249-55.

16. Kim HR, Kim DJ, Kang DR, Lee JG, Lim SM, Lee CY, et al. Fibroblast Growth Factor Receptor 1 Gene Amplification Is Associated With Poor Survival and Cigarette Smoking Dosage in Patients With Resected Squamous Cell Lung Cancer. Journal of Clinical Oncology 2013;31:731-7.

17. Majewski IJ, Mittempergher L, Davidson NM, Bosma A, Willems SM, Horlings HM, et al. Identification of recurrent FGFR3 fusion genes in lung cancer through kinome-centred RNA sequencing. J Pathol 2013;230:270-6.

18. Seo JS, Ju YS, Lee WC, Shin JY, Lee JK, Bleazard T, et al. The transcriptional landscape and mutational profile of lung adenocarcinoma. Genome Res 2012;22:2109-19.

19. Wu YM, Su F, Kalyana-Sundaram S, Khazanov N, Ateeq $\mathrm{B}$, Cao X, et al. Identification of targetable FGFR gene fusions in diverse cancers. Cancer Discov 2013;3:636-47.

20. Freier K, Schwaenen C, Sticht C, Flechtenmacher C, Muhling J, Hofele C, et al. Recurrent FGFR1 amplification and high FGFR1 protein expression in oral squamous cell carcinoma (OSCC). Oral Oncol 2007;43:60-6.

21. Gadgeel SM, Chen W, Cote ML, Bollig-Fischer A, Land $\mathrm{S}$, Schwartz AG, et al. Fibroblast growth factor receptor 1 amplification in non-small cell lung cancer by quantitative real-time PCR. PLoS One 2013;8:e79820.
22. Goke F, Bode M, Franzen A, Kirsten R, Goltz D, Goke A, et al. Fibroblast growth factor receptor 1 amplification is a common event in squamous cell carcinoma of the head and neck. Modern Pathology 2013;26:1298-306.

23. Goke F, Franzen A, Menon R, Goltz D, Kirsten R, Boehm $\mathrm{D}$, et al. Rationale for treatment of metastatic squamous cell carcinoma of the lung using fibroblast growth factor receptor inhibitors. Chest 2012;142:1020-6.

24. Heist RS, Mino-Kenudson M, Sequist LV, Tammireddy S, Morrissey L, Christiani DC, et al. FGFR1 amplification in squamous cell carcinoma of the lung. J Thorac Oncol 2012;7:1775-80.

25. Schildhaus HU, Heukamp LC, Merkelbach-Bruse S, Riesner K, Schmitz K, Binot E, et al. Definition of a fluorescence in-situ hybridization score identifies high- and low-level FGFR1 amplification types in squamous cell lung cancer. Mod Pathol 2012;25:1473-80.

26. Schultheis AM, Bos M, Schmitz K, Wilsberg L, Binot E, Wolf J, et al. Fibroblast growth factor receptor 1 (FGFR1) amplification is a potential therapeutic target in small-cell lung cancer. Mod Pathol 2013.

27. Tran TN, Selinger CI, Kohonen-Corish MRJ, McCaughan BC, Kennedy CW, O'Toole SA, et al. Fibroblast growth factor receptor 1 (FGFR1) copy number is an independent prognostic factor in non-small cell lung cancer. Lung Cancer 2013;81:462-7.

28. Weiss J, Sos ML, Seidel D, Peifer M, Zander T, Heuckmann JM, et al. Frequent and focal FGFR1 amplification associates with therapeutically tractable FGFR1 dependency in squamous cell lung cancer. Sci Transl Med 2010;2:62ra93.

29. Ishizuka T, Tanabe C, Sakamoto H, Aoyagi K, Maekawa M, Matsukura N, et al. Gene amplification profiling of esophageal squamous cell carcinomas by DNA array CGH. Biochem Biophys Res Commun 2002;296:152-5.

30. Loga KV, Kohlhaussen J, Marx AH, Sauter G, Grob T, Quaas A. FGFR1 amplification is linked to the squamous cell carcinoma subtype in esophageal carcinoma. . Cancer research 2013;73:abstr 3024.

31. Lecia V. Sequist, Phillippe Cassier, Andrea Varga, Josep Tabernero, Jan HM Schellens, Jean-Pierre Delord, et al. Phase I study of BGJ398, a selective pan-FGFR inhibitor in genetically preselected advanced solid tumors In: Lowe SW, editor. AACR Annual Meeting 2014; 2014; San Diego; 2014. p. CT326.

32. Andre F, Ranson M, Dean E, Varga A, Noll Rvd, Stockman PK, et al. Results of a phase I study of AZD4547, an inhibitor of fibroblast growth factor receptor (FGFR), in patients with advanced solid tumors. Cancer Res 2013;73:abstr LB-145.

33. Elbauomy Elsheikh S, Green AR, Lambros MB, Turner NC, Grainge MJ, Powe D, et al. FGFR1 amplification in breast carcinomas: a chromogenic in situ hybridisation analysis. Breast Cancer Res 2007;9:R23. 
34. Turner N, Pearson A, Sharpe R, Lambros M, Geyer F, Lopez-Garcia MA, et al. FGFR1 amplification drives endocrine therapy resistance and is a therapeutic target in breast cancer. Cancer Res 2010;70:2085-94.

35. Dutt A, Salvesen HB, Chen TH, Ramos AH, Onofrio RC, Hatton C, et al. Drug-sensitive FGFR2 mutations in endometrial carcinoma. Proc Natl Acad Sci U S A 2008;105:8713-7.

36. Cappellen D, De Oliveira C, Ricol D, de Medina S, Bourdin J, Sastre-Garau X, et al. Frequent activating mutations of FGFR3 in human bladder and cervix carcinomas. Nat Genet 1999;23:18-20.

37. Liao RG, Jung J, Tchaicha J, Wilkerson MD, Sivachenko A, Beauchamp EM, et al. Inhibitor-sensitive FGFR2 and FGFR3 mutations in lung squamous cell carcinoma. Cancer Res 2013;73:5195-205.

38. Couraud S, Zalcman G, Milleron B, Morin F, Souquet PJ. Lung cancer in never smokers - A review. European Journal of Cancer 2012;48:1299-311. 